\title{
Preface to the special issue on Polyoxometalates
}

\author{
Hong-Jin Lv ${ }^{1}(0)$ \\ Received: 1 January 2022 / Revised: 10 January 2022 / Accepted: 10 January 2022 / Published online: 25 January 2022 \\ (C) The Nonferrous Metals Society of China 2022
}

I feel deeply honored to be invited as the guest editor of this special issue on Polyoxometalates (POMs). POMs are a large family of early transition metal-oxo anionic clusters with atomically precise chemical composition and geometric structures formed by corner-, edge- and face-sharing of the $\mathrm{MO}_{6}(\mathrm{M}=\mathrm{Mo}, \mathrm{W}, \mathrm{V}, \mathrm{Nb}$, and Ta in high oxidation states) octahedral building blocks, which fully fulfills the aims and scope of the Tungsten journal launched in early 2019. The history of POM chemistry has been almost 200 years since the first reported example of polyoxometalate as an ammonium phosphomolybdate with the formula of $\left(\mathrm{NH}_{4}\right)_{3}\left[\mathrm{PMo}_{12} \mathrm{O}_{40}\right]$ by Berzelius in 1826. After that, great efforts have been devoted to solving and determining its geometrical structure, Linus Pauling was one of the first scientists trying to describe its structure, in terms of ionic radii. However, such structure was not unambiguously determined until the tungstate analogue $\left[\mathrm{PW}_{12} \mathrm{O}_{40}\right]^{3-}$ was reported in Nature 1933 by J. F. Keggin. Tremendously thanks to the advances in modern high-resolution, sophisticated instrumentation (e.g. single-crystal X-ray crystallography) as well as the diversity of synthetic and analytical methods, the POM research area has been extensively investigated. A serious of famous POM structures have been continuously reported, including the Anderson-Evans POM structure in 1948, the Wells-Dawson POM structure in 1953, the Lindqvist and Dexter-Silverton POM structures in 1968, the Strandberg-type POM structure in 1973, and the Müller big-molybdate-ball (Keplerate) structure in 1998, etc. In addition, the lacunary POM ligands generated by controllably removing one or several terminal $[\mathrm{W}=\mathrm{O}]^{4+}$ building blocks from the traditional plenary Keggin or Wells-Dawson POMs are very effective multidentate O-donor coordinating ligands to stabilize various mono- or multinuclear $\mathrm{d}$ and f-block transition metals, resulting in the isolation and

\section{Hong-Jin Lv}

hlv@bit.edu.cn

1 MOE Key Laboratory of Cluster Science, School of Chemistry and Chemical Engineering, Beijing Institute of Technology, Beijing 102488, China identification of numerous transition-metal-substituted POM structures since the mid twentieth century. The attractive physicochemical properties of POMs including rich redox chemistry, tunable compositions (size, charge density, acid strength, etc.), high thermal stability in the solid state, and solubility in polar/nonpolar solvents, have rendered POMs of great interest in various research fields such as catalysis, medicine, biology, photochemistry, magnetism, materials science, energy storage, and others.

The present special issue on POMs consists of eight contributions with one review paper and seven original research articles, providing an excellent overview of the current state as well as updates of the POM research field. The review paper by Zheng and co-workers summarized the recent advances of polyoxoniobate-catalyzed reactions, including decontamination of chemical warfare agents and organic dyes, epoxidation reactions, photocatalytic hydrogen evolution, and base-catalyzed reactions. The authors have also provided an outlook on the current challenges and perspectives of this research area. Among seven original research articles, three of them focused on the preparation of new POM-based catalyst for organic substrate transformation. For example, Han and co-workers reported the construction of Hydrogen bond-mediated $\left[\mathrm{SiW}_{12} \mathrm{O}_{40}\right]^{4-}$-based metalorganic networks and used it as efficient heterogeneous catalyst for selective oxidation of aryl alkenes to aldehydes. Zhang and co-workers synthesized two unprecedented peroxotantalum-containing clusters which exhibited excellent catalytic activity in catalyzing the amidation reactions of anhydrides and amines. Yang and co-workers reported the synthesis of a sandwich-type U(VI)-containing silicotungstates and investigated its catalytic activity for the synthesis of substituted phenylsulfonyl-1H-pyrazoles. Besides, the tri-vanadium-capped Keggin phosphomolybdate has been studied by both Chen and Ma lab, respectively. Chen and coworkers have studied the electrocatalytic activity for reduction of $\mathrm{H}_{2} \mathrm{O}_{2}$ and oxidization of AA as well as conspicuous catalytic degradation activity for $\mathrm{MB}, \mathrm{RhB}$, and $\mathrm{MO}$ under UV light. While Ma and co-workers have reported the construction of an electrochemical sensor for efficiently 
detection of L-cysteine. In addition, the application of POM in antibacterial area has also been illustrated by $\mathrm{Li}$ and co-workers, they have prepared a new organic-inorganic hybrid compound $\left[\mathrm{Co}(\mathrm{L})_{2}\right]_{2}\left[\mathrm{~W}_{6} \mathrm{O}_{19}\right](\mathrm{HL}=2$-acetylpyridine thiosemicarbazone) that exhibited excellent antibacterial activities superior to the clinical drugs (amoxicillin trihydrate, kanamycin sulfate). Finally, Lu and co-workers have explored two proton-conduction composites by encapsulating Keggin-type POM and organic amines into a sulfonated MIL-101 host which show high proton conductivity and high stability as promising proton-conduction materials.

I sincerely hope that this special issue on POMs could help attract a diverse readership of Tungsten journal, particularly to members of the following communities: polyoxometalates, photocatalysis, organic synthesis, and energy storage, etc. Also, more contributions from all aspects of POM chemistry are highly encouraged to be published in Tungsten journal in the near future. Last but not least, I would like to appreciate all the authors, reviewers, and publishing staff for their great efforts and contributions to the successful publication of this special issue.

$$
\text { Hongjin W }
$$

Hong-jin Lv

\section{Declarations}

Conflict of interest The authors declare no conflicts of interest.

Publisher's Note Springer Nature remains neutral with regard to jurisdictional claims in published maps and institutional affiliations.

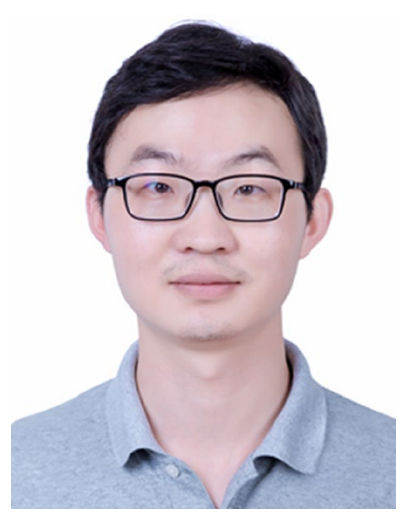

Dr. Hong-Jin Lv received his B.S. degree (2010) in Chemistry from Wuhan University and Ph.D. degree (2015) from Emory University, then worked as a postdoctoral researcher at University of Rochester from 2015 to 2018. In 2018, he joined the School of Chemistry and Chemical Engineering at Beijing Institute of Technology as a professor of Inorganic Chemistry. He has been awarded the Recruitment Program of Global Experts (Young Talents) and BIT Excellent Young Scholars Research Fund. His research interest is focused on designing structurally-new POMs and constructing POM-based functional materials for solar energy conversion/storage, value-added transformation of organic chemicals, and environmental remediation. 